November 8, 2019 13:24 WSPC/INSTRUCTION FILE "Time integration with balance relations"

\author{
International Journal of Structural Stability and Dynamics \\ (C) World Scientific Publishing Company \\ Preprint of an article published in International Journal of Structural Stability and Dynamics, 2020 DOI 10.1142/S0219455420500467 \\ ๑) 2020 World Scientific Publishing Co Pte Ltd https://www.worldscientific.com/doi/10.1142/S0219455420500467

\section{Improvement of discrete-mechanics-type time-integration schemes by utilizing balance relations in integral form together with Picard-type iterations} \\ Evgenii Oborin \\ Johannes Kepler University Linz, Altenbergerstraße 69, 4040 Linz, Austria \\ Hans Irschik \\ Johannes Kepler University Linz, Altenbergerstraße 69, 4040 Linz, Austria \\ hans.irschik@jku.at \\ Received (Day Month Year) \\ Accepted (Day Month Year) \\ The search for efficient explicit time integration schemes is a relevant topic in the current \\ literature on dynamic mechanical systems. In the present paper we describe a strategy of \\ utilizing the balance relations of mechanics in their integral form, so-called general laws \\ of balance, where the time-evolution of the integrands is approximated by established \\ computational techniques of the discrete-mechanics type. In a Picard-type iteration, the \\ outcomes are used for repeating the procedure several times, leading to an increased \\ accuracy. The advantages of the present explicit approach are discussed in the context \\ of linear and nonlinear motions of the mathematical pendulum. We utilize modern sym- \\ bolic procedures to obtain the time integration formulae and compare the results of our \\ methods with exact solutions and with the results of higher order implicit methods and \\ also with a recent explicit formulation from the literature. \\ Keywords: Discrete mechanics; Picard iteration; explicit time integration scheme; bal- \\ ance relations; symbolic computation; computer algebra

\section{Introduction} \\ The goal of the present contribution is to present a computational strategy for \\ the accurate and efficient numerical time integration of the equations of motion \\ of dynamic mechanical systems, based on the fundamental relations of balance in \\ their most general form, namely the form that involves integrals over time. \\ In the integral form, the relation of balance of some entity $\psi$ that is associated \\ with the mechanical system under consideration can be written as

$$
\psi(t)-\psi(0)=\int_{0}^{t} R(\tau) d \tau .
$$ \\ Balance relations of momentum, but also of energy and entropy for any mechanical \\ system can be put into a form that is consistent with (1.1). For details on this \\ integral form in the context of the classical field theories of mechanics, see Sect. 194
}


of Truesdell and Toupin [1], who referred to it as the general balance relation. For various examples in the mechanics of solids and fluids, containing the equations of motion of the single particle and the plane pendulum in integral form, see Ziegler [2]. The relation (1.1) can be extended to account for the presence of several non40 standard effects, such as balance of systems with a time-varying mass, see e.g. Irschik and Humer [3].

For instance, in case of balance of linear momentum for a single particle with a constant mass $m$, there is $\psi(t)=m v(t)$ in (1.1). The absolute velocity vector of the particle at time $t$ is $v(t)$, and $\psi(0)=m v(0)$ denotes the initial linear momentum. In case of balance of moment of momentum for the plane motion of a mathematical pendulum with mass $m$, a system that we will study subsequently for comparison sake, we have $\psi(t)=m L^{2} \omega(t)$, where the absolute angular velocity of the pendulum at time $t$ is denoted as $\omega(t)$, and $L$ is the distance between the fixed support and the center of gravity of the pendulum mass.

In (1.1), the resultant source that is responsible for the time-wise change of $\psi$, when written for the intermediate time instant $\tau$, is denoted as $R(\tau)$, where $0 \leq \tau \leq t$. This resultant source represents the instantaneous total of the influx, the supply, and the production quantities of $\psi$, see [1]. In the special case of balance of linear momentum of a single particle, $R$ stands for the resultant $F$ of the external forces that act upon the particle, while for balance of moment of momentum of a plane pendulum, $R$ represents the resulting moment $M$ of the external forces about the fixed support of the pendulum, see [2].

We consider the integral formulation stated in (1.1) as superior to the wellknown differential form of balance that can be associated with (1.1) by assuming the respective entities to be sufficiently smooth, resulting in an ordinary differential equation of first order. In contrast to the differential form, the integral form (1.1) can directly deal with discontinuous sources $R$. It also holds when Dirac-type singular functions are present in $R$. As pointed out in Sec. 194 of [1], relation (1.1) even holds, when there is a jump in $\psi$, where the integral in (1.1) then has to be understood in the sense of Stieltjes, cf. [1]. Correspondingly, the Stieltjes integral form of (1.1) has been called the fully general form of the relation of balance in [1]. The latter leads to a so-called impulsive balance, a situation, which however also will not be considered in the present contribution.

Important kinematic quantities can be computed from the time-evolution of $\psi$ by further time-wise integration. For instance, the instantaneous position vector $r$ of a single particle follows as

$$
r(t)-r(0)=\int_{0}^{t} v(\tau) d \tau .
$$

The initial position vector is $r(0)$. Analogously, for the plane pendulum, $r(t)$ is to be replaced by the instantaneous inclination angle $\varphi(t)$, measured from the vertical direction, say, where the initial angle is $\varphi(0)$. For the pendulum, one has to replace 
A main point in the present context of numerical time-initegration is that the integral form of the relation of balance (1.1), as well as the kinematic relation (1.2), already represent time-discrete formulations, which are at our disposal without having introduced any approximation. As a drawback for the usage in numerical time-integration, it however must be noted that the resultant source $R(\tau)$ in $(1.1)$ in general is a function of the yet unknown position $r(\tau)$ and velocity $v(\tau)$ of the system at time $\tau$. For instance, recall that the moment $M(\tau)$ of the weight of the pendulum about the fixed support depends on the angle $\varphi(\tau)$, see $[2]$ and the examples discussed below. Now, since position and velocity at time $\tau$ in general are not known in advance, the integrals in (1.1) and (1.2) in general cannot be evaluated directly, and, at a first glance, the latter relations appear to be of little use for the numerical time-integration of mechanical systems.

A strategy from the mathematical theory of ordinary differential equations, which dates back to Emile Picard, and which appears to be suitable for overcoming the above mentioned situation, is the Picard iteration, [4]. In short, substituting the initial condition into the integrand of the integral version of the differential equation of first order at hand as a trial solution, performing the integration and repeating the procedure with the result of this integration as a new trial function, leads to a suitable successive approximation technique. Differential equations of higher orders are solved as a number of simultaneous equations, each of the first order. For some more recent presentations containing concise considerations on existence, convergence and error-bounds, see Chapter 7 of the book by Hirsh, Smale and Devaney [5], Chapter 1.8 of the book by Hairer, Nørsett and Wanner [6], or Chapter 8 of the book by Agarwal and O'Regan [7]. An alternative to the original Picard iteration was presented by Djang [8], who suggested to start the iteration with any convenient function that satisfies the initial conditions, in order to improve convergence of the successive approximation.

It is the goal of the present contribution to specify the suggestion by Djang [8] by utilizing as a first guess well-known numerical schemes that were formulated for the sake of approximately integrating (1.1) or its differential counterparts. Our hypothesis is that, applying these already quite powerful numerical schemes for the intermediate time-instant $\tau$ and substituting the corresponding positions and velocities into the source $R(\tau)$ in (1.1), the numerical error at the time $t$ will be strongly decreased by the integration. The same is supposed to hold for the case (1.2), when substituting the result of the procedure for (1.1) into the integral in (1.2), but applying the procedure for (1.1) at the intermediate time $\tau$, resulting in $v(\tau)$. The whole technique can be repeated several times, in consecutive iterations, leading to higher-order schemes that are supposed to further improve the accuracy of the results at time $t$. Afterwards, one may proceed to the next time step, using the results of the first time step at time $t$ as initial values for the second time step. Particularly when the problem at hand is formulated as an autonomous one, 
4 Hans Irschik and Evgenii Oborin

i.e., when it is translation-invariant, see, e.g., Chapter 2 of the book by Hirsch, Smale and Devaney [5], this should lead to a powerful time-stepping method. It is emphasized that the development is particularly facilitated by modern symbolic computation methods.

It is also pointed out that, as will be discussed later in more detail, the suggested procedure does guarantee that the relation of balance of kinetic energy, often denoted as the theorem of power expended, [1], or shortly called the power law, [2], is satisfied in an algorithmic sense. The reason is that the relation of balance of kinetic energy is a consequence of balance of momentum, see e.g. [2] and [3]. To the opinion of the present authors, algorithmic satisfaction of the power law is an important feature; however it does not mean that, in case of conservative mechanical systems, for which a potential of the sources $R$ does exist, the requirement of a conservation of the total of the kinetic and the potential energy is satisfied in an exact sense by the approximate solutions.

In the following, we exemplarily demonstrate the validity of our hypothesis, i.e. that improvements can be gained through the repeated usage of the integral balance relation (1.1) in the sense of the Picard iteration, but starting with the established numerical time-integration schemes from the literature as a first guess. We particularly study the motion of the linear and the nonlinear pendulum, for which analytical solutions do exist for the sake of comparison, and which is frequently used in the literature as a benchmark-test. Since we wish to remain as simple as possible, we subsequently use explicit time-integration schemes from the literature for starting the first iteration step.

Our paper is organized as follows: In Sect. 2 below, we introduce some explicit time-integration schemes from the literature, which then will be used in order to start the Picard-type iteration procedure. For other explicit time-integration schemes that could be used, the reader is referred to [9] and [10]. Here, we first shortly address two highly valuable explicit time-integration schemes, which were presented by Greenspan in [11], at which occasion he introduced the notion of discrete mechanics. Subsequently, these two discrete mechanics formulations will be referred to as G1orig and G2orig, respectively. A modification of G2orig using the mid-point rule subsequently will also be studied and will be referred to as G2Morig. In the course of time, Greenspan and coworkers presented also various implicit time integration schemes, which not only became influential in the field of physics, but which also can be considered as role models for so-called conservative or structure preserving time integrators in nonlinear structural mechanics, see, e. g., [12-15]. A list of the important contributions of Greenspan can be found in his book on particle modeling [16]. For further implicit time integration techniques, see, e. g., Kim and Reddy [17]. The latter reference will be used subsequently for comparison sake also, likewise to the implicit formulation by Fung [14]. In Sect. 2 below, we also shortly introduce a recent explicit method by Kim and Lee [18], later referred to as KLorig, as well as the explicit fourth-order Runge-Kutta scheme, abbreviated as RK4orig. See, e. g., Chapter 2 of the book by Hairer, Nørsett and Wanner [6] 

is interesting to note that an equivalence of the explicit Runge-Kutta scheme and the original iteration was established by Gur'ianov [19]. Following [19], the solution of the problem by means of the $n$th order Runge-Kutta method is the same as it follows from the original Picard method after $n$ iterations. An estimation of the error of the Runge-Kutta method was presented in [19] based on this connection.

In Sect. 3 below, the explicit time-integration techniques mentioned above exemplarily are applied as a first guess in the Picard-type iteration for computing the motion of the linearized mathematical pendulum, for which an analytic solution in terms of trigonometric functions exists. A single time step with arbitrary initial conditions is studied in order to compare the accuracy of the resulting five approximation schemes, denoted as G1, G2, G2M, KL and RK4, respectively. The errors in the inclination angle and in the total energy, as well as the spectral radii of the provided solutions are presented at the end of the dimensionless time step after four Picard-type iterations. Moreover, a comparison of computational costs of the different approaches is provided. It can be seen that the suggested Picard-type procedures lead to substantial improvements with respect to the accuracy, as well as with respect to the computational costs, since a large number of time steps is needed for the original procedures in order to reach the same accuracy as the novel Picard-type formulations.

Section 4 then delas with the motion of the non-linear mathematical pendulum, considering a periodic motion with insufficient energy for swinging over, for which an exact solution in terms of Jacoby elliptic functions is at disposal. Periodic motions with a large number of periods are considered using multiple time steps and a comparatively large time-step length. Since the mechanical system under consideration is autonomous, the Picard-type iterations for the five schemes can be performed once, before the time-stepping procedure begins. This is an essential feature with respect to computational efficiency. We note that non-autonomous systems under certain conditions may be rewritten into homogeneous systems, see [5]. For the non-linear pendulum, the integrands in (1) and (2) contain non-linear functions. For a convenient integration, the non-linear terms are expanded into Taylor series, and integration is performed by means of symbolic computation. The number of terms that is taken into account in the Taylor series of course does influence the accuracy at the end of the time step, likewise to the number of Picard-type iterations. An exemplary study with respect to a single time step is presented for the five novel methods G1, G2, G2M, KL and RK4, respectively. Afterwards, a multistep study is performed for the pendulum with large initial angles and over a large number of periods of motion. While the single-step study demonstrates that quite good accuracy can be reached with smaller numbers, particularly for G2M, KL and RK4, the multi-step computations are exemplarily performed using fourteen terms in the Taylor series and four Picard-type iterations, since the computational costs are quite small in comparison to the original versions of the five methods, which need substantially smaller time steps for reaching similar orders of accuracy. 
6 Hans Irschik and Evgenii Oborin

A study on accuracy in comparison to results from the literature is also successfully performed. The paper ends with concluding remarks containing an outlook on future work.

\section{Some explicit time integration schemes for starting the Picard-type iteration}

The two explicit time integration schemes introduced by Greenspan in [11] were written for the motion of a single particle, and they are shortly repeated subsequently. The first explicit scheme reads, when applied to the time instant $\tau$ :

$$
\begin{gathered}
m(v(\tau)-v(0))=F(0) \tau, \\
r(\tau)-r(0)=\frac{v(\tau)+v(0)}{2} \tau,
\end{gathered}
$$

which gives

$$
\begin{gathered}
v(\tau)=v(0)+\frac{F(0)}{m} \tau, \\
r(\tau)=r(0)+v(0) \tau+\frac{F(0)}{m} \frac{\tau^{2}}{2} .
\end{gathered}
$$

The initial resultant force is written as $F(0)$. Note that (2.4) also follows when substituting (2.1) into (1.2). The scheme outline will be further denoted by G1orig.

An extended scheme has been suggested in Greenspan [11] (G2orig is its reference below), in which the mid-point approximation (2.2) is retained, but in which (2.3) is extended. When applied for the time instant $\tau$, this second scheme by Greenspan [11] reads

$$
v(\tau)=v_{1}+\left(\frac{3}{2} F_{1}-\frac{1}{2} F(0)\right) \frac{1}{m} \frac{\tau}{2} .
$$

Here, $F_{1}$ means the resultant force at time $\tau / 2$ corresponding to the arguments

$$
\begin{gathered}
v_{1}=v(0)+\frac{F(0)}{m} \frac{\tau}{2}, \\
r_{1}=r(0)+\frac{\tau}{4}\left(v_{1}+v(0)\right)=r(0)+v(0) \frac{\tau}{2}+\frac{F(0)}{m} \frac{\tau^{2}}{8}
\end{gathered}
$$

for velocity and position, respectively, see formulae (4.1) and (4.2) of [11].

To approximate the position vector at time $\tau$, the estimate originally suggested by Greenspan [11] gives

$$
r(\tau)=r_{1}+\frac{\tau}{4}\left(v_{1}+v(\tau)\right)=r(0)+v(0) \tau+\frac{F(0)}{m} \frac{5 \tau^{2}}{16}+\frac{F_{1}}{m} \frac{3 \tau^{2}}{16} .
$$


We may use the mid-point approximation rule (2.2) together with (2.5) and (2.6) (G2Morig)

$$
r(\tau)=r(0)+\frac{v(0)+v(\tau)}{2} \tau=r(0)+v(0) \tau+\frac{F(0)}{m} \frac{\tau^{2}}{8}+\frac{F_{1}}{m} \frac{3 \tau^{2}}{8} .
$$

We may also build our numerical method on the basis of the fourth-order RungeKutta method and the recent explicit method of Kim and Lee [18] (the scheme with no dissipation appears to be the most accurate for the considered examples and is used in the paper). (The original versions will be further called RKorig and KLorig.) The Kim-Lee formula leads to

$$
r(\tau)=\frac{T^{2}}{4 m}\left(F_{\tau_{1}}+F_{\tau_{2}}\right)+T v(0),
$$

where $F_{\tau_{1}}$ and $F_{\tau_{2}}$ mean the resultant force at time $\tau_{1}$ and $\tau_{2}$ with the position and velocity given by

$$
\begin{gathered}
r_{\tau_{1}}=r(0)+\frac{T}{4} v(0), \\
v_{\tau_{1}}=v(0), \\
r_{\tau_{2}}=\frac{T^{2}}{8} \frac{F_{\tau_{1}}}{m}+r(0)+\frac{T}{2} v(0), \\
v_{\tau_{2}}=\frac{T}{2} \frac{F_{\tau_{1}}}{m}+v(0) .
\end{gathered}
$$

Following our hypothesis described above, the four explicit schemes presented in (2.1)-(2.12) and the fourth-order Runge-Kutta scheme, subsequently abbreviated as RK4orig, now will be applied as starting points for developing novel schemes that are based on the integral formulations stated in (1.1) and (1.2), using a Picard-type iteration. For the formulas necessary to set up RK4orig, see [20]. The displacements and velocity approximations in (2.1)-(2.12) are substituted into the source $R(\tau)$ in (1.1), which here represents the resulting force $F(\tau)$ that acts upon the single particle, and the integral in (1.1) is eventually solved, obtaining a first new estimate for $\psi(t)=m v(t)$. This is facilitated because the estimates in (2.1)-(2.12) enter the specific functional form of $F(\tau)$ via the initial value $F(0)$ only, which is a known entity. In order to proceed further, time $t$ is replaced by $\tau$ in (1.1), and the corresponding function $v(\tau)$ is substituted into (1.2) in order to obtain a first new estimate for the displacement $r(t)$ by integration. Alternatively, the mid-point approximation, see (2.2), may be used. Replacing again time $t$ by $\tau$, we obtain $r(\tau)$, which, together with $v(\tau)$, gives a new estimate for $R(\tau)=F(\tau)$ in (1.1). The procedure thus can be repeated a second time, where a second novel estimate is obtained for $v(t)$, and the corresponding $v(\tau)$ can be substituted into (1.2) giving a second novel estimate for $r(t)$. Proceeding further, a single time-step formulation with $n$ iterative steps is obtained, where it is supposed that the $n$th iteration gives estimates that are more accurate than the $(n-1)$ th one. Utilizing the results of the $n$th repetition of $r(t)$ and $v(t)$ as initial values, one can proceed to a second time 
step, $m=2$. Performing analytic integrations, the formulas for $v(\tau)$ and $r(\tau)$ from the $n$th repetition of the first time step can be used directly, so that no formula updates then are to be performed in the second and in the following time steps, up to the final time step with number $m=N$.

This procedure will be subsequently applied to the motion of the plane mathematical pendulum, replacing the force $F$ by the moment $M$, and the velocity $v$ by the angular velocity $\omega$, etc. The linearized equation of motion of the pendulum will be treated in Sect. 3 below, while the nonlinear pendulum will be discussed in Sect. 4. Our results presented below are based on $n=4$ repetitions of the Picard type that are performed before the time-stepping procedure starts (recall that the corresponding labels of the novel Picard-type schemes are G1, G2, KL, RK4, and G2M).

\section{Linearized pendulum equation}

In order to demonstrate advantages of our above suggested strategy, namely to volve and utilize (1.1) and (1.2), we first consider small pendulum oscillations with respect to a stable equilibrium point. The restoring moment is linearized as follows:

$$
M(\tau)=-m g L \varphi(\tau)
$$

The exact solution to the linearized problem is known to be, see [2],

$$
\varphi(t)=\varphi(0) \cos \Omega t+\frac{\omega(0)}{\Omega} \sin \Omega t, \quad \Omega=\sqrt{\frac{g}{L}} .
$$

We start with Greenspan's first scheme (G1orig), see (2.3) and (2.4). From (2.4), one obtains with $M(0)=-m g L \varphi(0)$ that

$$
\varphi(\tau)=\varphi(0)\left(1-\frac{(\Omega \tau)^{2}}{2}\right)+\omega(0) \tau .
$$

Substituting the Greenspan discrete mechanics estimate (3.3) into (3.1) and integrating according to (1.1) and (1.2) lead to the following novel estimates:

$$
\begin{gathered}
\omega(t)=\Omega \varphi(0)\left(-\Omega t+\frac{(\Omega t)^{3}}{6}\right)+\omega(0)\left(1-\frac{(\Omega t)^{2}}{2}\right), \\
\varphi(t)=\varphi(0)\left(1-\frac{(\Omega t)^{2}}{2}+\frac{(\Omega t)^{4}}{24}\right)+\frac{\omega(0)}{\Omega}\left(\Omega t-\frac{(\Omega t)^{3}}{6}\right) .
\end{gathered}
$$

Indeed, we find by comparison that the new formulation in (3.4) and (3.5) provides an improvement in the Taylor expansion of the exact solution (3.2) by one term in comparison to the Greenspan formula (3.3). We repeat the substitution and integration and find that the $n$th iteration of this procedure reproduces $2 n+2$ terms of the Taylor expansion of the exact solution. This result appears to be already quite outstanding. 
Improvement of discrete-mechanics-type time-integration schemes with balance relations... 9

We use (3.4) and (3.5) to demonstrate exemplarily that this method (and the following novel procedures) leads to an algorithmic satisfaction of the power law. Substituting the Greenspan estimate (3.3) into (3.1), we obtain

$$
M(\tau)=-m g L\left(\varphi(0)\left(1-\frac{(\Omega \tau)^{2}}{2}\right)+\omega(0) \tau\right) .
$$

In order to obtain (3.4), this moment has been substituted into the balance relation (1.1) and the integration has been performed. Now, in the framework of our algorithm, the power of $M(\tau)$ becomes

$$
P(\tau)=M(\tau) \omega(\tau)
$$

with the novel result $\omega(\tau)$ according to (3.4). Setting $R(\tau)=P(\tau)$ in (1.1) and performing the integration, it turns out that

$$
\psi(t)-\psi(0)=\frac{m L^{2}}{2} \omega(t)^{2}-\frac{m L^{2}}{2} \omega(0)^{2}
$$

with $\omega(t)$ from (3.4). This result indeed can be identified as the difference of the instantaneous and initial kinetic energy of the pendulum, see [2], when using the new estimate (3.4) for $\omega(t)$. Hence, balance of kinetic energy, or the power law, is satisfied in an algorithmic sense. This is because balance of kinetic energy can be considered as a mathematical consequence of balance of momentum, see [2] and [3] for general derivations. Note that the power law remains satisfied also for the higher-order iterations, since balance of momentum is utilized in each of them. This is also true for the schemes starting with the Greenspan estimates (2.5)-(2.9).

We now proceed to the two schemes (G2 and G2M) based on the second scheme in Greenspan [11], see (2.5)-(2.9), to the Kim-Lee scheme (KL) (2.10), and to the fourth-order Runge-Kutta scheme (RK). For the moment in (3.1), we obtain with (2.7) that

$$
M_{1}=-m g L \varphi_{1}=-m g L \varphi(0)-m g L \omega(0) \frac{\tau}{2}-M(0) \frac{(\Omega \tau)^{2}}{8} .
$$

Hence, using $M(0)=-m g L \varphi(0),(2.5)$ becomes

$$
\omega(\tau)=-\varphi(0) \Omega\left(\Omega \tau-\frac{3(\Omega \tau)^{3}}{32}\right)+\omega(0)\left(1-\frac{3(\Omega \tau)^{2}}{8}\right) .
$$

The original Greenspan approximation, G2orig, (2.8) then gives

$$
\varphi(\tau)=\varphi(0)\left(1-\frac{(\Omega \tau)^{2}}{2}+\frac{(\Omega \tau)^{4}}{128}\right)+\frac{\omega(0)}{\Omega}\left(\Omega \tau-\frac{3(\Omega \tau)^{3}}{32}\right) .
$$

The mid-point approximation, G2Morig, (2.9) yields

$$
\varphi(\tau)=\varphi(0)\left(1-\frac{(\Omega \tau)^{2}}{2}+\frac{3(\Omega \tau)^{4}}{64}\right)+\frac{\omega(0)}{\Omega}\left(\Omega \tau-\frac{3(\Omega \tau)^{3}}{16}\right) .
$$



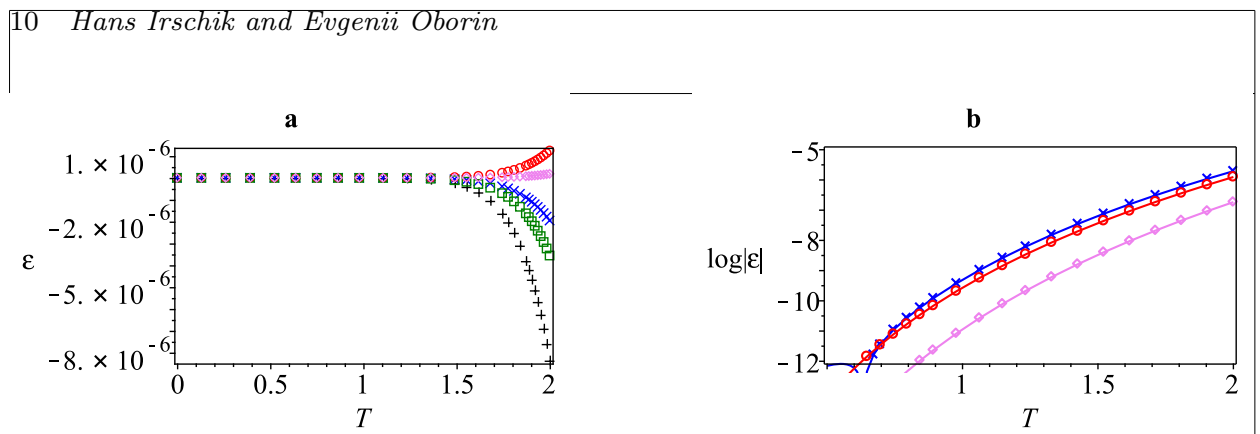

Fig. 1. Relative error in angle for $\omega(0)=0, \varphi(0)=1$ : G1 (black crosses), G2 (green boxes), KL (blue diagonal crosses), G2M (red circles), and RK (violet diamonds).

The Kim-Lee formula (2.10) with no dissipation, KLorig, [18] follows to

$$
\varphi(\tau)=\varphi(0)\left(1-\frac{(\Omega \tau)^{2}}{2}+\frac{(\Omega \tau)^{4}}{64}\right)+\frac{\omega(0)}{\Omega}\left(\Omega \tau-\frac{3(\Omega \tau)^{3}}{16}+\frac{(\Omega \tau)^{5}}{128}\right) .
$$
plus potential energy) for these approaches, where we substitute the approximations

The relations in (3.11)-(3.13) are used as starting estimates. Substituting, e.g., (3.12) into (3.1) and integrating according to (1.1) and (1.2) lead to the first estimates

$$
\begin{aligned}
& \omega(t)=\Omega \varphi(0)\left(-\Omega t+\frac{(\Omega t)^{3}}{6}-\frac{3(\Omega t)^{5}}{320}\right)+\omega(0)\left(1-\frac{(\Omega t)^{2}}{2}+\frac{3(\Omega t)^{4}}{64}\right), \\
& \varphi(t)=\varphi(0)\left(1-\frac{(\Omega t)^{2}}{2}+\frac{(\Omega t)^{4}}{24}-\frac{(\Omega t)^{6}}{640}\right)+\frac{\omega(0)}{\Omega}\left(\Omega t-\frac{(\Omega t)^{3}}{6}+\frac{3(\Omega t)^{5}}{320}\right) .
\end{aligned}
$$

It is seen that this scheme leads to a further improvement, compare (3.4) and (3.5), which however does not coincide with the next higher terms in the Taylor expansion of the exact solution. The same is true when starting with (3.11) or (3.13).

In Fig. 1 we show the relative error of the five approximate schemes (G1, G2, KL, G2M, and RK) at the end of first time step and for four iterations, $n=4$. The error is computed as

$$
\varepsilon=\frac{\varphi_{\text {approx }}-\varphi_{\text {exact }}}{\varphi(0)}
$$

where $T=\Omega t$ is the dimensionless time-step length. The pendulum is assumed to start from $\varphi(0)=1$ at rest, $\omega(0)=0$. Everywhere in this paper we use the symbolic computer code Maple with 24 significant digits. An excellent degree of accuracy can be observed from Fig. 1; e. g., in case of $T=\pi / 2$, the magnitude of the relative error is in the range from $4.6 \times 10^{-7}$ to $6.3 \times 10^{-9}$.

Since the mechanical system under consideration, the mathematical pendulum, is conservative, we also present the error in conservation of total energy (kinetic 
November 8, 2019 13:24 WSPC/INSTRUCTION FILE "Time integration with balance relations"

$$
\begin{aligned}
& \text { Improvement of discrete-mechanics-type time-integration schemes with balance } \\
& \text { into the exact formula, see }[2] \text {, which yields: } \\
& \qquad \varepsilon=\frac{\varphi_{\text {approx }}^{2}+\left(\frac{\omega_{\text {approx }}}{\Omega}\right)^{2}-\left(\varphi^{2}(0)+\left(\frac{\omega(0)}{\Omega}\right)^{2}\right)}{\varphi^{2}(0)+\left(\frac{\omega(0)}{\Omega}\right)^{2}}
\end{aligned}
$$

In Fig. 2 the case $\omega(0)=0, \varphi(0)=1$ is treated. It turns out that the error is comparatively small, and the method based on the Runge-Kutta scheme (RK) leads to superior results. Note that theses methods differ also by the number of used elementary computations. The comparison is given in Table 1, where RK scheme is most advantageous.
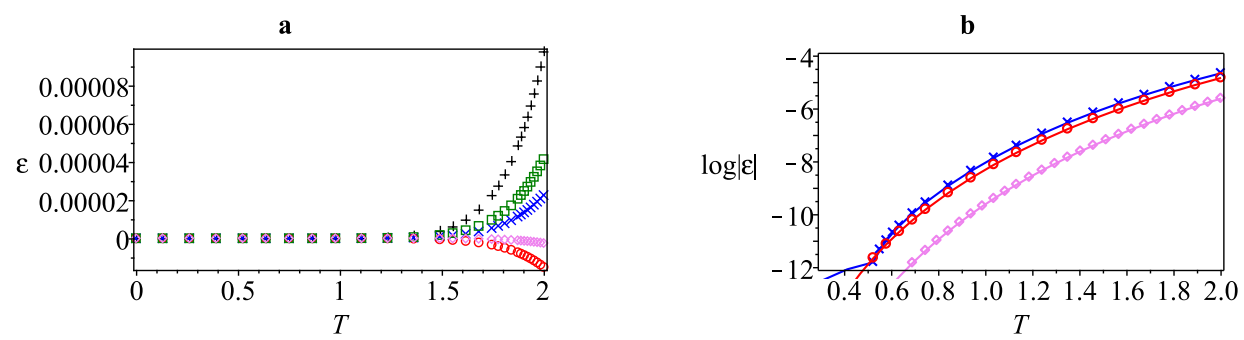

Fig. 2. Error in energy conservation $\omega(0)=0, \varphi(0)=1$ : G1 (black crosses), G2 (green boxes) KL (blue diagonal crosses), G2M (red circles), and RK (violet diamonds).

Table 1. Comparison of computational cost of different approaches in the linear case.

\begin{tabular}{lccc}
\hline Method & No. additions & No. multiplications & No. timesteps \\
\hline G1 & 10 & 64 & 1 \\
KL & 12 & 89 & 1 \\
G2M & 14 & 118 & 1 \\
RK & 14 & 118 & 1 \\
G1orig & 2 & 4 & 1 \\
KLorig & 7 & 19 & 1 \\
G1orig* & $>1000000$ & $>2000000$ & $>500000$ \\
KLorig* & 193900 & 526300 & 27700 \\
\hline
\end{tabular}

Note: ${ }^{*}$ To reach the accuracy of RK at $T=1$.

The last step of investigating the linear problem will be a study on the stability.

Following the representation by Hughes [21], we evaluate here the spectral radii $\rho$ of the solutions provided above. The results are shown in Fig. 3. Following [21], we should require that $\rho \leq 1$.

It turns out that the stablest method with respect to initial conditions is the G2M, as long as $T \leq 1.903$. The RK scheme is stable until 1.854 , and the KL

scheme is stable until 1.457 . 


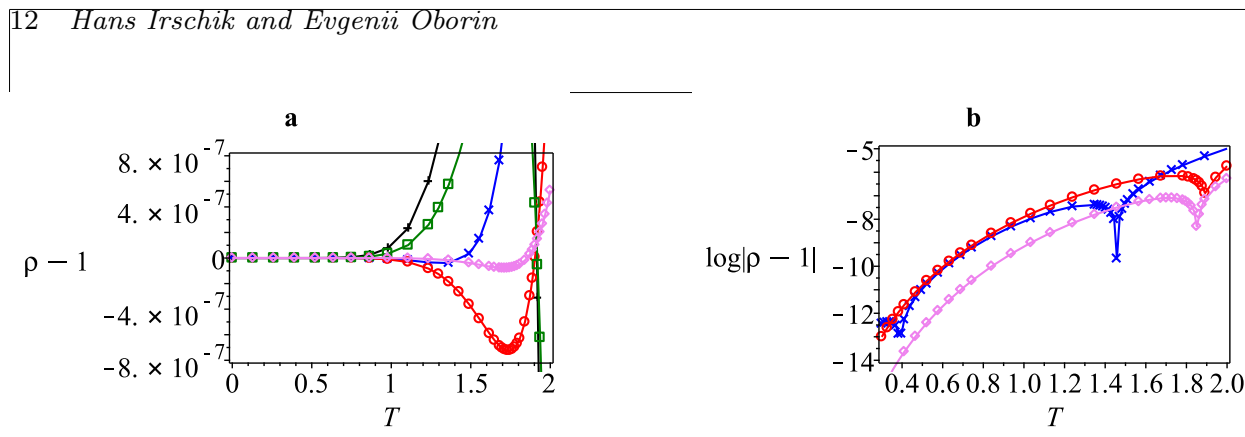

Fig. 3. Spectral radii: G1 (black crosses), G2 (green boxes), KL (blue diagonal crosses), G2M (red circles), and RK (violet diamonds).

We also outline the possible directions of the algorithm optimization to achieve better cost/efficiency ratio: we compare the results for different numbers of Picardtype iterations, see Table 2.

Table 2. Effect of number of Picard-type iterations for different approaches in the linear case: the angle error is computed with (3.16).

\begin{tabular}{lccccc}
\hline Method & 0 iter. & 1 iter. & 2 iter. & 3 iter. & 4 iter. \\
\hline G1 & -0.584 & 0.0828 & $-0.608 \cdot 10^{-2}$ & $0.274 \cdot 10^{-3}$ & $-0.837 \cdot 10^{-5}$ \\
KL & -0.0838 & 0.0161 & $-0.131 \cdot 10^{-2}$ & $0.622 \cdot 10^{-4}$ & $-0.195 \cdot 10^{-5}$ \\
G2M & 0.166 & -0.0172 & $0.107 \cdot 10^{-2}$ & $-0.436 \cdot 10^{-4}$ & $0.125 \cdot 10^{-5}$ \\
RK & 0.0828 & $-0.608 \cdot 10^{-2}$ & $0.274 \cdot 10^{-3}$ & $-0.837 \cdot 10^{-5}$ & $0.185 \cdot 10^{-6}$ \\
\hline
\end{tabular}

Concluding the present linear section, we can state that the suggested procedure leads to substantial improvements with respect to the accuracy of the approximations. Starting from discrete mechanics schemes presented in [11], from the wellknown Runge-Kutta scheme, and from the novel Kim-Lee scheme and using four iterations, i. e., four repeated applications of the balance law (1.1), the obtained high degree of accuracy is demonstrated in Fig. 1 for a single time step, $m=1$, $n=4$, and $t=T$. All of the schemes do satisfy the power law in an algorithmic sense, but the total energy is not exactly conserved, see Figs. 2 and 3. As can be seen, however, the errors in energy conservation are comparatively small. Conditional stability according to the spectral radius requirement of the G2M, KL, and RK schemes could be proved, while stability is absent for the other schemes.

In connection with the high degree of accuracy, it can be supposed, however, that the solution procedures will work also after a large number of time steps. This will be demonstrated for the nonlinear pendulum in the next section. 
Improvement of discrete-mechanics-type time-integration schemes with balance relations... 13

\section{Nonlinear pendulum}

\subsection{Single time step}

Consider the plane motion of a mathematical pendulum in the field of gravity, now utilizing the following exact nonlinear expression for the restoring moment,

$$
M(t)=-m g L \sin \varphi(t) .
$$

For a periodic motion with insufficient energy for swinging over, the exact solution of the corresponding local relation of balance of angular momentum,

$$
\ddot{\varphi}=-\frac{g}{L} \sin \varphi
$$

is, see e. g. [2] and [22]:

$$
\begin{aligned}
& \theta(t)=\operatorname{sgn}(\dot{\varphi}(0)) \Omega t+\operatorname{sn}^{-1}\left(\kappa k_{0} \mid k\right), \\
& \varphi(t)=2 \arcsin (k \operatorname{sn}(\theta(t) \mid k))
\end{aligned}
$$

The notation $\operatorname{sn}(\ldots)$ means the Jacobi elliptic sine, and $\operatorname{sgn}(\ldots)$ is the signum function. The following abbreviations are used: $\Omega^{2}=g / L, k^{2}=E_{0} / E_{p}, \kappa=1 / k$, $k_{0}=\sin (\varphi(0) / 2), E_{0}=\dot{\varphi}(0)^{2}+E_{p} k_{0}^{2}, E_{p}=4 \Omega^{2}$.

For the sake of numerical approximation, we start the proposed schemes by determining the angular velocity and the angle according to the formulae (2.6) and $(2.7)$ :

$$
\begin{aligned}
\omega_{1} & =\omega(0)-\Omega^{2} \sin \varphi(0) \frac{\tau}{2}, \\
\varphi_{1} & =\varphi(0)+\frac{\tau}{4}\left(\omega_{1}+\omega(0)\right) .
\end{aligned}
$$

Here $\tau$ is an intermediate time instant. Taking Eq. (4.4) into consideration and following Eq. (2.5), we obtain

$$
\omega=\omega_{1}+\Omega^{2}\left(-\frac{3}{2} \sin \varphi_{1}+\frac{1}{2} \sin \varphi(0)\right) \frac{\tau}{2} .
$$

The determination of the angle are different for the methods studied here (the conditionally stable method and the most accurate one, see Sec. 3 for modeling of a linear pendulum). For the mid-point approximation method we have

$$
\begin{array}{r}
\varphi=\varphi(0)+\omega(0) \tau-\frac{5}{16} \Omega^{2} \sin \varphi(0) \tau^{2}- \\
-\frac{3}{16} \Omega^{2} \sin \left(\varphi(0)+\frac{1}{2} \omega(0) \tau-\frac{1}{8} \Omega^{2} \sin \varphi(0) \tau^{2}\right) \tau^{2} .
\end{array}
$$

Similarly, we may write the formulas for the Kim-Lee (2.10) and fourth-order Runge-Kutta schemes.

We now apply (1.1) by analogy in order to obtain an estimate for the angular velocity. Instead of using numerical integration, we expand expressions for $\sin \varphi$ in the moment, see (4.1), that result from (4.6), into their Taylor series, in order to simplify the integration of (1.1). The number of expansion terms taken into account 
14 Hans Irschik and Evgenii Oborin

in the Taylor series approximation of course influences the accuracy of the scheme. Subsequently, we will present results for using 14 terms in the Taylor expansions. The computations can be easily performed symbolically, e. g. by using the Maple procedures taylor and int. The angles at the end of the time step then follow either from the mid-point rule, from the Kim-Lee, or from the fourth-order RungeKutta schemes. As in the linear case in Sec. 3, we repeat this procedure according to (1.1) and (1.2) four times, as a basis for the subsequent time-stepping procedure. As above, the schemes are referred to as G1, G2, KL, G2M, and RK.

\subsection{Multiple time steps}

In order to compare the outcomes of our suggested explicit methodology with the exact solution presented in [2] and [22], as well as with the outcomes of powerful recent implicit numerical procedures, as presented by Kim \& Reddy in [17] (KR), and by Fung in [23] (F), we apply the above derived single step formulae in the framework of a multiple time step procedure. For direct comparison with the numerical solutions in [17] and [23], we set $\Omega=1 \mathrm{~s}^{-1}$ and take the initial velocity as $\omega(0)=1.999999238 \mathrm{~s}^{-1}$, which is used in [17] and [23]. The outcomes of the different computations are shown in Table 3 for the time instant $t=T_{f}$, which is the quarter of the period of oscillation of the nonlinear pendulum. We note that, in our hands, the exact solution obtained by Maple yields $T_{f}=8.430254948 \mathrm{~s}$, which is slightly different from the value $T_{f}=8.430255141 \mathrm{~s}$ mentioned in [17]. However, the dicrepancy in results because of this difference is negligible. Having subdivided $T_{f}$ into various time steps $m$, in Table 3 we show (i) the exact values as derived by Maple using 24 digits, (ii) the results of the higher order implicit schemes presented in [17] and [23], (iii) the results of the two explicit formulations originally presented by Greenspan [11] (G1orig and G2orig), as well as (iv) the results of the present explicit procedures according to formulae (4.6), Kim-Lee formula (2.10), and fourth-order Runge-Kutta scheme (G2M, KL, and RK). For the meanings of the respective orders of the implicit methods and the parameter $\mu$, the reader is referred to [17] and [23]. It is seen that the formulations in [11] fail to give adequate results, even for a larger number of time steps, such as for $m=100$. For $m=25$, the present explicit formulations are one order more accurate than the high-order implicit formulations reported in [17] and [23]; for larger time steps, $m=10$ and $m=25$, the implicit schemes in [17] and [23] are more accurate.

415 Using the same nonlinear example, we also compare the errors when the different number of Taylor terms is considered. Table 4 presents the results for the RungeKutta method with the different number of iterations.

It may be useful to compare the schemes where an increasing number of Taylor terms is used for the subsequent iterations. Table 5 shows the corresponding results with the number of Taylor terms different at each iteration. We denote one term in the first iteration step, two in the second one, three in the third one, and four in the fourth one by by $1,2,3,4$. 
November 8, 2019 13:24 WSPC/INSTRUCTION FILE "Time integration with balance relations"

\begin{tabular}{|c|c|c|c|}
\hline \multicolumn{4}{|c|}{ Improvement of discrete-mechanics-type time-integration schemes with balance relations... } \\
\hline Method & $m=\frac{T_{f}}{\Delta t}$ & $\varphi\left(T_{f}\right)$ & Error $=\frac{\text { exact }-\varphi\left(T_{f}\right)}{\text { exact }}$ \\
\hline Exact & & 3.139847324 & \\
\hline $\mathrm{KR}(7$ th-order, $\mu=0),[17]$ & 25 & 3.139833891 & $0.427840 \times 10^{-5}$ \\
\hline KR (8th-order, $\mu=1),[17]$ & 25 & 3.139846872 & $0.144169 \times 10^{-6}$ \\
\hline KR (10th-order, $\mu=1),[17]$ & 10 & 3.139848406 & $0.344411 \times 10^{-6}$ \\
\hline $\mathrm{F}(7$ th-order, $\mu=0),[23]$ & 25 & 3.139834046 & $0.422900 \times 10^{-5}$ \\
\hline $\mathrm{F}(8$ th-order, $\mu=1),[23]$ & 25 & 3.139847686 & $-0.115138 \times 10^{-6}$ \\
\hline F (10th-order, $\mu=1),[23]$ & 10 & 3.139846547 & $-0.247731 \times 10^{-6}$ \\
\hline G1orig, [11, Eqs. (1.1)-(1.3)] & 100 & 9.113077815 & $1.902395 \times 10^{0}$ \\
\hline G2orig, [11, Eqs. (4.1) and (4.2)] & 100 & 9.240294283 & $1.942912 \times 10^{0}$ \\
\hline \multirow[t]{3}{*}{$\mathrm{KL}$} & 10 & 3.049865606 & $-0.286580 \times 10^{-1}$ \\
\hline & 15 & 3.139744704 & $-0.326832 \times 10^{-4}$ \\
\hline & 25 & 3.139846781 & $-1.726790 \times 10^{-7}$ \\
\hline \multirow[t]{3}{*}{ RK } & 10 & 3.049882950 & $-0.286524 \times 10^{-1}$ \\
\hline & 15 & 3.139744797 & $-0.326534 \times 10^{-4}$ \\
\hline & 25 & 3.139846794 & $-1.687329 \times 10^{-7}$ \\
\hline \multirow[t]{3}{*}{ G2M } & 10 & 3.049865394 & $-0.286580 \times 10^{-1}$ \\
\hline & 15 & 3.139744717 & $-0.326789 \times 10^{-4}$ \\
\hline & 25 & 3.139846793 & $-1.689897 \times 10^{-7}$ \\
\hline
\end{tabular}

Table 4. Comparison of nonlinear numerical solutions at $T_{f}=8.430254948 \mathrm{~s}$ for different numbers of Taylor terms in highly nonlinear case $\left(\omega(0)=1.999999238 \mathrm{~s}^{-1} ; \varphi_{\max }=3.139847324, m=\frac{T_{f}}{\Delta t}=25\right)$ : Error $=\frac{\text { exact }-\varphi\left(T_{f}\right)}{\text { exact }}$ with RK method.

\begin{tabular}{lcccc}
\hline No. terms & 1 It. & 2 It. & 3 It. & 4 It. \\
& & & & \\
4 & $3.75 \cdot 10^{-1}$ & $3.75 \cdot 10^{-1}$ & $3.75 \cdot 10^{-1}$ & $3.75 \cdot 10^{-1}$ \\
6 & $-8.82 \cdot 10^{-3}$ & $-1.19 \cdot 10^{-2}$ & $-1.19 \cdot 10^{-2}$ & $-1.19 \cdot 10^{-2}$ \\
7 & $5.36 \cdot 10^{-4}$ & $-1.91 \cdot 10^{-3}$ & $-1.91 \cdot 10^{-3}$ & $-1.91 \cdot 10^{-3}$ \\
8 & $2.38 \cdot 10^{-3}$ & $3.67 \cdot 10^{-4}$ & $3.73 \cdot 10^{-4}$ & $3.73 \cdot 10^{-4}$ \\
9 & $2.18 \cdot 10^{-3}$ & $8.44 \cdot 10^{-5}$ & $8.93 \cdot 10^{-5}$ & $8.93 \cdot 10^{-5}$ \\
10 & $2.11 \cdot 10^{-3}$ & $-1.48 \cdot 10^{-5}$ & $-1.13 \cdot 10^{-5}$ & $-1.13 \cdot 10^{-5}$ \\
12 & $2.12 \cdot 10^{-3}$ & $-3.78 \cdot 10^{-6}$ & $1.11 \cdot 10^{-7}$ & $1.08 \cdot 10^{-7}$ \\
14 & $2.12 \cdot 10^{-3}$ & $-4.03 \cdot 10^{-6}$ & $-1.65 \cdot 10^{-7}$ & $-1.69 \cdot 10^{-7}$ \\
16 & $2.12 \cdot 10^{-3}$ & $-4.03 \cdot 10^{-6}$ & $-1.63 \cdot 10^{-7}$ & $-1.67 \cdot 10^{-7}$ \\
\hline
\end{tabular}

To compare the results of the proposed methods with the exact solution for a longer observation period, the errors in conservation of the total energy are presented first. For simplicity we use a zero initial condition $\omega(0)=0$. The relative energy error of the proposed solutions can be computed using the following formula, which becomes zero for the exact solution, compare [2]:

$$
\varepsilon=\frac{1-\cos \varphi+\frac{\omega^{2}}{2 \Omega^{2}}}{1-\cos \varphi(0)}-1 .
$$


November 8, 2019 13:24 WSPC/INSTRUCTION FILE "Time integration with balance relations"

\begin{tabular}{|c|c|c|c|c|c|}
\hline \multicolumn{6}{|c|}{$\begin{array}{l}\text { Table 5. Comparison of nonlinear numerical solutions at } T_{f}=8.430254948 \mathrm{~s} \text { for } \\
\text { different numbers of Taylor terms in highly nonlinear case }(\omega(0)=1.999999238 \mathrm{~s}-1 \\
\left.\varphi_{\max }=3.139847324, m=\frac{T_{f}}{\Delta t}=25\right): \text { Error }=\frac{\text { exact }-\varphi\left(T_{f}\right)}{\text { exact }} \text { with RK method. }\end{array}$} \\
\hline No. & No. terms & $1 \mathrm{It}$. & 2 It. & 3 It. & $4 \mathrm{It}$. \\
\hline 0 & $14,14,14,14$ & $2.12 \cdot 10^{-3}$ & $-4.03 \cdot 10^{-6}$ & $-1.65 \cdot 10^{-7}$ & $-1.69 \cdot 10^{-7}$ \\
\hline 1 & $10,12,14,14$ & $2.11 \cdot 10^{-3}$ & $-3.78 \cdot 10^{-6}$ & $-1.65 \cdot 10^{-7}$ & $-1.69 \cdot 10^{-7}$ \\
\hline 2 & $10,12,12,14$ & $2.11 \cdot 10^{-3}$ & $-3.78 \cdot 10^{-6}$ & $1.11 \cdot 10^{-7}$ & $-1.69 \cdot 10^{-7}$ \\
\hline 3 & $10,10,12,14$ & $2.11 \cdot 10^{-3}$ & $-1.48 \cdot 10^{-5}$ & $1.11 \cdot 10^{-7}$ & $-1.69 \cdot 10^{-7}$ \\
\hline 4 & $8,10,12,14$ & $2.38 \cdot 10^{-3}$ & $-1.48 \cdot 10^{-5}$ & $1.11 \cdot 10^{-7}$ & $-1.69 \cdot 10^{-7}$ \\
\hline 5 & $6,8,10,14$ & $-8.83 \cdot 10^{-3}$ & $3.67 \cdot 10^{-4}$ & $-1.13 \cdot 10^{-5}$ & $-1.61 \cdot 10^{-7}$ \\
\hline 6 & $6,6,10,14$ & $-8.83 \cdot 10^{-3}$ & $-1.19 \cdot 10^{-2}$ & $4.10 \cdot 10^{-6}$ & $-1.67 \cdot 10^{-7}$ \\
\hline 7 & $4,6,10,14$ & $3.75 \cdot 10^{-1}$ & $-1.19 \cdot 10^{-2}$ & $4.10 \cdot 10^{-6}$ & $-1.67 \cdot 10^{-7}$ \\
\hline 8 & $4,6,10,12$ & $3.75 \cdot 10^{-1}$ & $-1.19 \cdot 10^{-2}$ & $4.10 \cdot 10^{-6}$ & $1.02 \cdot 10^{-7}$ \\
\hline 9 & $4,6,10,10$ & $3.75 \cdot 10^{-1}$ & $-1.19 \cdot 10^{-2}$ & $4.10 \cdot 10^{-6}$ & $-1.13 \cdot 10^{-5}$ \\
\hline 10 & $4,6,8,12$ & $3.75 \cdot 10^{-1}$ & $-1.19 \cdot 10^{-2}$ & $3.73 \cdot 10^{-4}$ & $-2.55 \cdot 10^{-7}$ \\
\hline 11 & $6,6,8,10$ & $-8.83 \cdot 10^{-3}$ & $-1.19 \cdot 10^{-2}$ & $3.73 \cdot 10^{-4}$ & $-1.13 \cdot 10^{-5}$ \\
\hline 12 & $6,6,6,12$ & $-8.83 \cdot 10^{-3}$ & $-1.19 \cdot 10^{-2}$ & $-1.19 \cdot 10^{-2}$ & $1.34 \cdot 10^{-5}$ \\
\hline 13 & $4,4,10,12$ & $3.75 \cdot 10^{-1}$ & $3.75 \cdot 10^{-1}$ & $-5.37 \cdot 10^{-4}$ & $3.68 \cdot 10^{-7}$ \\
\hline 14 & $2,6,10,12$ & $-1.84 \cdot 10^{0}$ & $4.31 \cdot 10^{-3}$ & $-2.85 \cdot 10^{-5}$ & $1.35 \cdot 10^{-7}$ \\
\hline
\end{tabular}

We show this relative error $\varepsilon$ in Fig. 4 for the comparatively large number of 50 periods of oscillations, using $m=10000$ time steps. In the first case, see Fig. 4a, we treat a large initial angle equal to $\varphi(0)=3.139847324$, i. e. nearly vertical initial position. In the second case, see Fig. $4 \mathrm{~b}$, we study an initial inclination of $\varphi(0)=3 \pi / 4$. It can be seen that the error remains small in both cases and for both methods, the RK scheme being superior.

In Fig. 5 we show the phase trajectories of the two considered cases, $\varphi(0)=$ 3.139847324 and $\varphi(0)=3 \pi / 4$. It turns out that the results of the two proposed methods are practically indistinguishable (they are also practically identical to the exact phase portraits). Most important, the solutions remain at the same phaseplane trajectory during the whole 50 periods of observation.
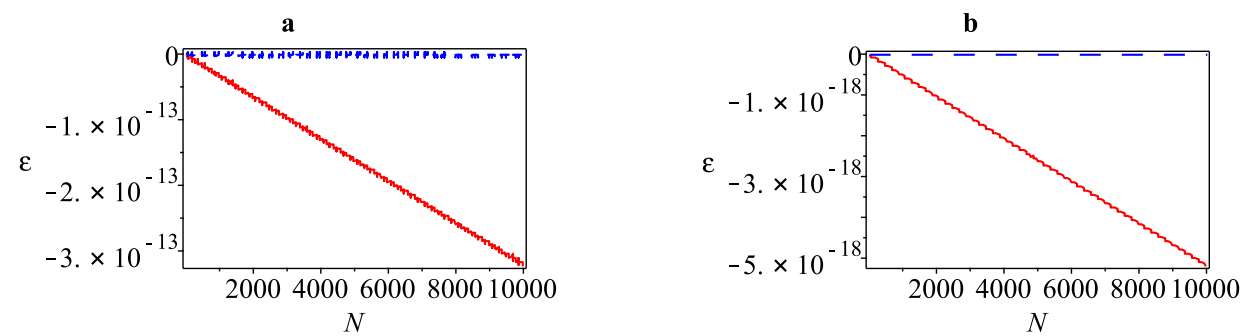

Fig. 4. Relative error in energy vs number of time steps; 50 periods of oscillations, 200 time steps per period: (a) $\varphi(0)=3.139847324$; (b) $\varphi(0)=3 \pi / 4$. RK scheme (blue dashed line) and G2M (red solid line). 
November 8, 2019 13:24 WSPC/INSTRUCTION FILE "Time integration with balance relations"

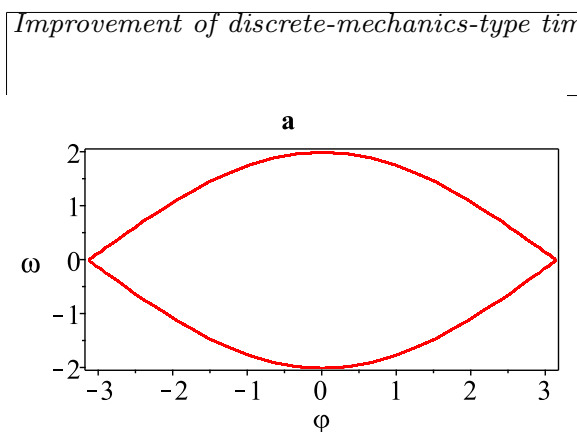

$\omega$

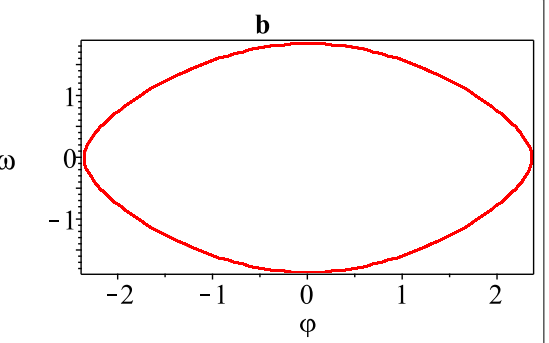

Fig. 5. Phase plane representation; 50 periods of oscillations, 200 time steps per period: (a) $\varphi(0)=3.139847324$; (b) $\varphi(0)=3 \pi / 4$. Phase trajectories turn out to be indistinguishable for both the RK and G2M schemes.

After that we treat the angle over time steps in Figs. 6 and 7. Here we use formula (3.16) to obtain the results for the relative error $\varepsilon$. As shown in Figs. 4 and 6 , the angle error is more pronounced than the error in energy conservation, but it remains comparatively small, even after 50 periods of nonlinear motion.

We performed the computations for a number of cases and found that in the vast majority the mid-point approximation scheme provides better accuracy with a less computational effort. The methods differ by number of elementary computations, see Table 6 , where the mid-point approximation formula is most advantageous.

Table 6. One-step computational cost of different approaches in the nonlinear case.

\begin{tabular}{lccc}
\hline Method & No. additions & No. multiplications & No. functions \\
\hline KL & 187 & 1110 & 14 \\
G2M & 113 & 898 & 14 \\
RK & 113 & 898 & 14 \\
KLorig & 7 & 18 & 3 \\
G2Morig & 5 & 10 & 3 \\
RKorig & 7 & 17 & 4 \\
\hline
\end{tabular}

We also have compared our formulation with an explicit time-stepping method
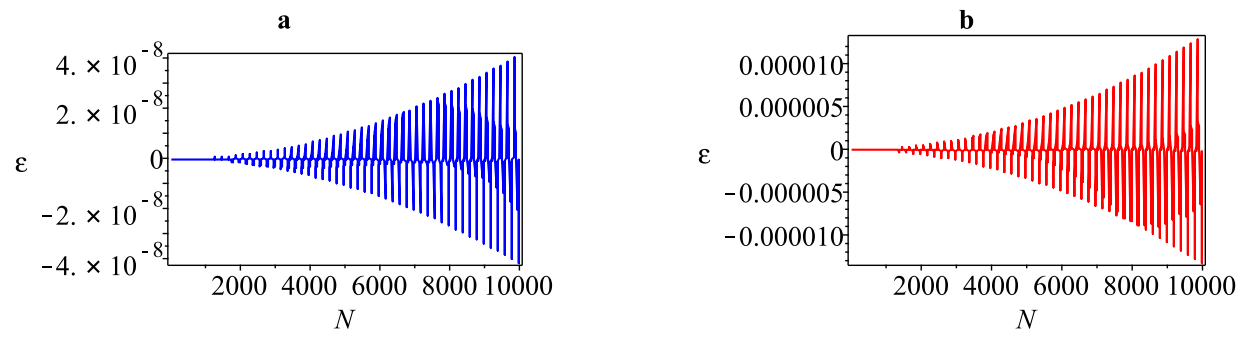

Fig. 6. Angle error for $\varphi(0)=3.139847324$ during 50 periods of oscillations (200 times steps per period): (a) RK and (b) G2M schemes. 


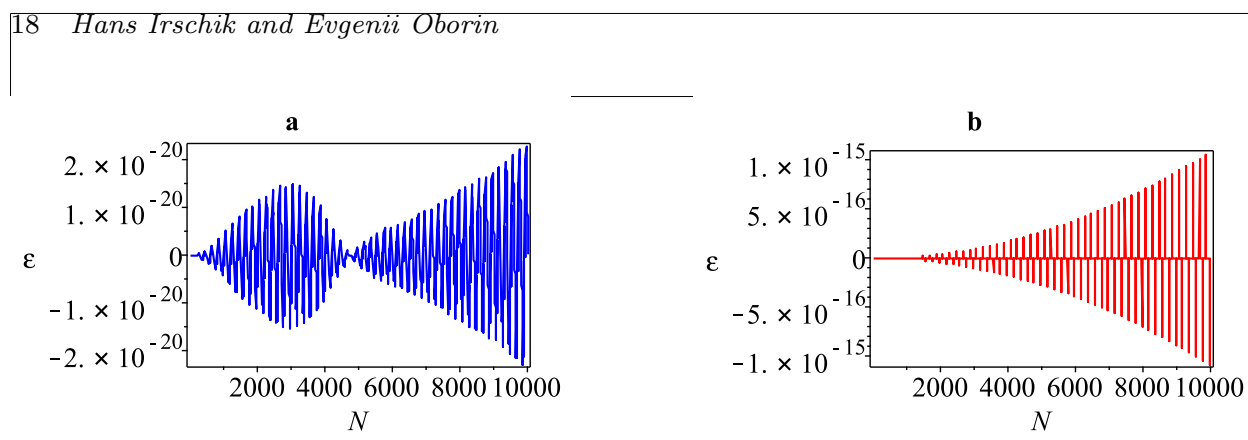

Fig. 7. Angle error for $\varphi(0)=3 \pi / 4$ during 50 periods of oscillations (200 time steps per period) (a) RK and (b) G2M schemes.

that has been recently suggested for nonlinear problems by Kim and Lee [18]. By comparison to Figs. 6-11 of [18], it has turned out for the oscillating pendulum that the present explicit method is able to yield a comparative accuracy with substantially larger time steps.

In concluding this section, we can state that the proposed explicit methods yield accurate results also for highly nonlinear pendulum motions and during long observation periods, the computational effort being comparatively low; the errors in energy conservation turn out to be very small; the errors in the angle being also comparatively small, even after 50 periods of nonlinear motion. The solutions remain on the same phase-plane trajectories during the whole observation period and practically do coincide with the exact phase-plane curves.

\section{Conclusions}

The main conclusions of the present paper are shortly summarized as follows:

- The general relations of balance (the time-discrete integral form of the relations of balance) has been involved in time integration of dynamical systems.

- Explicit numerical integration schemes from the literature, such as discrete mechanics schemes, have been taken as starting points for solving the integrals, and the procedure has been improved by repeated substitution, resulting in accurate and computationally efficient explicit approximate integration schemes.

- Powerful symbolic computer codes can be involved in a straightforward manner.

- Linear and nonlinear exact solutions for the plane pendulum motion have been exemplarily used for comparison sake, as well as with results of implicit higher-order formulations and a recent explicit numerical method from the literature.

- Improved accuracy also at quite large time steps and during large observation periods has been obtained. 
November 8, 2019 13:24 WSPC/INSTRUCTION FILE "Time integration with balance relations"

• Extensions considering multiple degrees of freedom are on the way.

Acknowledgement

This work has been supported by the COMET-K2 Center of the Linz Center

of Mechatronics (LCM) funded by the Austrian federal government and the federal state of Upper Austria.

\section{References}

1. C. Truesdell and R. Toupin, The classical field theories, in Principles of Classical Mechanics and Field Theory / Prinzipien der Klassischen Mechanik und Feldtheorie, S. Flügge, ed., Springer Berlin Heidelberg, Berlin, Heidelberg, 1960, pp. 226-858.

2. F. Ziegler, Mechanics of Solids and Fluids, 2nd Ed., Springer, Wien, 1995.

3. H. Irschik and A. Humer, A rational treatment of the relations of balance for mechanical systems with a time-variable mass and other non-classical supplies, in Dynamics of Mechanical Systems with Variable Mass, H. Irschik and A.K. Belyaev, eds., Springer, Vienna, 2014, pp. 1-50.

4. E. Picard, Sur l'application des methodes d'approximations successives a l'etude de certaines equations differentielles ordinaires, J. de Math. Pures et Appl., 4e serie 9 (1893), pp. 217-271.

5. M.W. Hirsch, S. Smale, and R.L. Devaney, Differential Eqautions, Dynamical Systems, and an Introduction to Chaos, 3rd. ed., Academic Press, Boston, 2013.

6. E. Hairer, S.P. Nørsett, and G. Wanner, Solving Ordinary Differential Equations I, Nonstiff Problems. Second revised edition, corrected 3rd printing, SpringerVerlag, Berlin Heidelberg, 2008.

7. R.P. Agarwal and D. O'Regan, An Introduction to Ordinary Differential Equations, Springer, New York, 2008.

8. G.F. Djang, A modified method of iteration of the Picard type in the solution of differential equations, Journal of the Franklin Institute 246 (1948), pp. 453-457.

9. S.Y. Chang, Comparisons of structure-dependent explicit methods for time integration, Int. J. Struct. Stability and Dynamics 15 (2015), p. 1450055.

10. M. Rezaiee-Pajand and M. Karimi-Rad, A new explicit time integration scheme for nonlinear dynamic analysis, Int. J. Struct. Stability and Dynamics 16 (2016), p. 1550054.

11. D. Greenspan, A new explicit discrete mechanics with applications, Journal of the Franklin Institute 294 (1972), pp. 231-240.

12. R.A. LaBudde and D. Greenspan, Energy and momentum conserving methods of arbitrary order for the numerical integration of equations of motion, Numerische Mathematik 25 (1975), pp. 323-346.

13. R.A. LaBudde and D. Greenspan, Energy and momentum conserving methods of arbitrary order for the numerical integration of equations of motion, $\mathrm{Nu}-$ merische Mathematik 26 (1976), pp. 1-16.

14. T. Hughes, T. Caughey, and W. Liu, Finite-element methods for nonlinear 
November 8, $2019 \quad$ 13:24 WSPC/INSTRUCTION FILE "Time integration with balance relations"

$20 \quad$ REFERENCES

elastodynamics which conserve energy, ASME J. Appl. Mech. 45 (1978), pp. 366-370.

15. P. Betsch, Structure-preserving Integrators in Nonlinear Structural Dynamics and Flexible Multibody Dynamics, Springer, Cham, 2016.

16. D. Greenspan, Particle Modeling, 3rd Edition, Birkhäuser, Boston, 2012.

17. W. Kim and J. Reddy, A new family of higher-order time integration algorithms for the analysis of structural dynamics, ASME J. Appl. Mech. 84 (2017), p. 071008.

18. W. Kim and J.H. Lee, An improved explicit time integration method for linear and nonlinear structural dynamics, Computers and Structures 206 (2018), pp. $42-53$.

19. V.M. Gur'ianov, On the connection between the Runge-Kutta method and Picard's method, J. Appl. Math. Mech. 28 (1964), pp. 783-786.

20. J.C. Butcher, Numerical methods for ordinary differential equations, 3rd Ed. Wiley, 2016.

21. T.J.R. Hughes, The Finite Element Method: Linear Static and Dynamic Finite Element Analysis, Prentice-Hall, Inc., Englewood Cliffs, New Jersey, 1987.

22. K. Ochs, A comprehensive analytical solution of the nonlinear pendulum, European Journal of Physics 32 (2011), pp. 479-490.

23. T.C. Fung, Solving initial value problems by differential quadrature method-part 2: second-and higher-order equations, International Journal for Numerical Methods in Engineering 50 (2001), pp. 1429-1454. 